\title{
Monocyte/HDL ratio in women with polycystic ovary syndrome and healthy controls
}

\author{
๑T)Tuğba Gürbüzz ${ }^{1}$, @Nefise Tanrıdan Okçu², @Nur Dokuzeylül Güngör ${ }^{3}$ \\ ${ }^{1}$ Medistate Hospital, Department of Gynecology and Obstetrics, İstanbul, Turkey \\ ${ }^{2}$ Adana City Research and Training Hospital, Department of Gynecology and Obstetrics, Adana, Turkey \\ ${ }^{3}$ Bahçeșehir University Göztepe Medical Park Hospital, Department of Gynecology and IVF Clinic, İstanbul, Turkey
}

Cite this article as: Gürbüz T, Tanrıdan Okçu N, Dokuzeylül Güngör N. Monocyte/HDL ratio in women with polycystic ovary syndrome and healthy controls. Anatolian Curr Med J 2021; 3(2); 98-103.

\begin{abstract}
Aim: To examine and compare various variables, especially monocyte/high-density lipoprotein (HDL) ratio (MHR), in women with polycystic ovary syndrome (PCOS) and healthy controls.

Material and Method: Data of patients who applied to Adana City Research and Training Hospital, Gynecology and Obstetrics Outpatient Clinic were analyzed retrospectively from the hospital database. The records of a total of 259 cases, including 194 with PCOS and 65 without PCOS who had normal menstrual cycles between the ages of 18-38, were examined.

Results: When compared with the control group, the overweight/obese PCOS group was found to have higher triglyceride value. Age and neutrophil values were significantly higher, whereas red blood cell distribution width was lower in the control group compared to both PCOS groups. Weight and body mass index values were significantly different for all three groups. The HDL value was detected to be significantly higher in the normoweight PCOS group compared to the overweight/obese PCOS group. Total cholesterol value was significantly higher in the overweight/obese PCOS group compared to the normoweight PCOS group. The MHR values was found to be similar in all groups.

Conclusions: Although the present study has various limitations, there are few studies on this subject and our findings represent an important difference from available results, suggesting the presence of underlying variations that necessitate further studies on this subject.
\end{abstract}

Keywords: Polycystic ovary syndrome, obesity, inflammation, monocyte, high-density lipoprotein

\section{INTRODUCTION}

Polycystic ovary syndrome (PCOS), one of the most common diseases among women, is a complex endocrine abnormality. It affects about $6-15 \%$ of all women of reproductive age $(1,2)$. In recent studies, researchers have suggested that the effects of PCOS are not limited to reproductive functions, with studies demonstrating significant influences on metabolism and the cardiovascular system (3). Insulin resistance is an important pathological indicator of both PCOS and cardiometabolic syndrome. The cellular and molecular mechanisms of insulin resistance in PCOS are still not fully understood $(4,5)$.

Many studies have examined various variables that play a role in the etiopathogenesis of PCOS. Although it is still unclear exactly what the underlying cause is, it has been suggested that imbalance at the level of luteinizing hormone and follicle-stimulating hormone, genetic factors and various environmental facilitators play important roles in the pathophysiology of PCOS (6-8). In addition to these factors, studies investigating the relationship between inflammation and PCOS have shown that there was a significant increase in almost all inflammatory markers in patients with PCOS $(9,10)$. Although it is not clear whether the inflammation detected in PCOS cases is the cause or result of the disease, it is clear that chronic lowgrade inflammation occurs in PCOS (10). The presence and severity of inflammation can be determined by various laboratory tests. Macrophages in tissues and monocytes in circulation contribute to inflammation. Increased monocyte activation is considered to be an indicator of 
increased inflammation (11). High-density lipoprotein (HDL) is involved in anti-inflammatory and antioxidant effects by preventing oxidation of low-density lipoprotein (LDL), altering the migration of macrophages, and activating monocytes. Therefore, while HDL increase suppresses inflammation, its decrease can be considered to be pro-inflammatory $(12,13)$.

Researchers have recently reported that the ratio of monocyte count to HDL (MHR) can be used as an inflammatory index (14-16). There are only a few studies evaluating the role of MHR in PCOS patients (17-19). Therefore, in the present study, we aimed to evaluate women with PCOS who did not have any additional health problems (in two different groups based on body mass index (BMI)) and to compare MHR values in these groups with a control group of healthy subjects. In addition, we aimed to investigate biochemical parameters that demonstrated differences in the examined population.

\section{MATERIAL AND METHOD}

Ethics committee approval was obtained from the Ethics Clinical Researches Committee of Adana City Training and Research Hospital (Approval no: 878, Date: 2020/05.20). Informed consent was obtained from all individual participants included in the study. The trial was conducted in accordance with the Helsinki Declaration principles. Data of patients who applied to the Gynecology and Obstetrics Outpatient Clinic of Adana City Training and Research Hospital were analyzed retrospectively, with data drawn from the hospital database. The records of a total of 259 cases, including 194 with PCOS (103 overweight/obese, 91 normoweight) and 65 patients without PCOS (and with normal menstrual cycles) between the ages of 18-38, were examined.

Women aged between 18-38 years who were diagnosed with PCOS, were non-smokers, and did not have any additional diseases were included in the study. Pregnant women, smokers, women in early menopause, those in lactation, and women with hypertension, diabetes mellitus, autoimmune diseases, chronic infectious diseases or adrenal gland disease were excluded from the study. In addition to the absence of any other clinical cause leading to hyperandrogenism, PCOS was diagnosed based on the Rotterdam consensus criteria with at least two of the following three criterions: chronic oligomenorrhea (having an annual menstrual count of six or fewer), biochemical hyperandrogenism (defined as elevation in serum total testosterone concentration), polycystic ovarian morphology in clinical evaluation and pelvic ultrasonography (detection of 10 or more cysts of $0.8-1.2 \mathrm{~cm}$ in circumference in both ovaries) (7). Cases with regular menstrual cycles and serum total testosterone levels within normal limits who did not have polycystic morphology in pelvic ultrasonography examination were included in the control group with regard to age and BMI.

\section{Statistical Analysis}

All analyses were performed on SPSS v21 (SPSS Inc., Chicago, IL, USA). For the normality check, the Kolmogorov-Smirnov test was used. Data are given as mean \pm standard deviation or median (1st quartile - 3rd quartile) for continuous variables according to normality of distribution. Normally distributed variables were analyzed with the ANOVA test between groups, while non-normally distributed variables were analyzed with the Kruskal-Wallis test. Bonferroni correction was employed in post-hoc comparisons. $\mathrm{P}<0.05$ values were accepted as statistically significant results.

\section{RESULTS}

While there was no statistically significant difference in terms of age between the two groups with PCOS, the control group had significantly higher age $(\mathrm{p}<0.01)$. When compared with the control group, the overweight/ obese PCOS group was found to have higher triglyceride value $(p<0.05)$. Age and neutrophil values were significantly higher, whereas red blood cell distribution width (RDW) was lower in the control group compared to both PCOS groups. Weight and BMI values were found to be significantly different for all 3 groups $(p<0.001)$. HDL value was detected to be significantly higher in normoweight PCOS patients than the overweight/obese PCOS group $(\mathrm{P}<0.01)$. The total cholesterol value was significantly higher in the overweight/obese PCOS group compared to the normoweight PCOS group. The MHR value was found to be similar in all groups (Table 1).

We performed multiple linear regression analysis to determine factors significantly effective on monocyte count. None of the variables included in the model, age $(\mathrm{p}=0.189)$, BMI $(\mathrm{p}=0.626)$, triglyceride $(\mathrm{p}=0.551)$, HDL ( $\mathrm{p}=0.614)$, total cholesterol $(\mathrm{p}=0.366)$, neutrophil $(\mathrm{p}=0.118)$ are RDW $(\mathrm{p}=0.283)$, were found to be significant (Table 2).

We performed multiple linear regression analysis to determine factors significantly effective on HDL value. We found that lower BMI was associated with higher monocyte level (OR: -0.617, 95\%CI: -0.833--0.108). Other variables included in the model, age $(\mathrm{p}=0.698)$, triglyceride $(\mathrm{p}=0.162)$, total cholesterol $(\mathrm{p}=0.860)$, neutrophil $(\mathrm{p}=0.375)$, monocyte $(\mathrm{p}=0.614)$ and $\mathrm{RDW}$ $(\mathrm{p}=0.112)$ were found be non-significant (Table 3$)$. 


\begin{tabular}{|c|c|c|c|c|}
\hline \multicolumn{5}{|c|}{ Groups } \\
\hline & Control & PCOS $(\mathrm{BMI}<25)$ & $\operatorname{PCOS}(\mathrm{BMI} \geq 25)$ & $\mathbf{p}$ \\
\hline $\mathrm{N}$ & 65 & 91 & 103 & N/A \\
\hline Age (years) & $31.80 \pm 5.58^{\mathrm{a}}$ & $29.77 \pm 4.40^{\mathrm{b}}$ & $29.35 \pm 4.72^{\mathrm{b}}$ & 0.005 \\
\hline Height (cm) & $166.25 \pm 6.06$ & $166.70 \pm 6.08$ & $166.67 \pm 7.44$ & 0.899 \\
\hline Weight (kg) & $66(60-72)^{\mathrm{a}}$ & $62(57-65)^{\mathrm{b}}$ & $86(80-96)^{c}$ & $<0.001$ \\
\hline BMI $\left(\mathrm{kg} / \mathrm{m}^{2}\right)$ & $23.81(21.61-26.37)^{\mathrm{a}}$ & $22.14(20.94-23.51)^{\mathrm{b}}$ & $30.85(27.72-34.37)^{c}$ & $<0.001$ \\
\hline Triglyceride (mg/dL) & $96(65-147)^{\mathrm{a}}$ & $120(88-148)^{\mathrm{ab}}$ & $126(96-153)^{\mathrm{b}}$ & 0.014 \\
\hline LDL (mg/dL) & $132(76-152)$ & $142(108-164)$ & $142(115-162)$ & 0.054 \\
\hline HDL (mg/dL) & $64(53-75)^{\mathrm{ab}}$ & $72(56-82)^{\mathrm{a}}$ & $62(48-74)^{\mathrm{b}}$ & 0.001 \\
\hline Total Cholesterol (mg/dL) & $204.02 \pm 57.42^{\mathrm{ab}}$ & $185.52 \pm 50.56^{\mathrm{a}}$ & $217.56 \pm 58.28^{b}$ & $<0.001$ \\
\hline White blood cell $(\times 1000)$ & $7.55(6.29-9.71)$ & $7.30(6.22-9.47)$ & $6.87(6.14-8.90)$ & 0.189 \\
\hline Neutrophil $(\times 1000)$ & $4.82(3.61-7.88)^{\mathrm{a}}$ & $3.94(3.19-5.82)^{\mathrm{b}}$ & $4.07(3.21-5.68)^{\mathrm{b}}$ & 0.030 \\
\hline Lymphocyte (×1000) & $2.25 \pm 0.81$ & $2.09 \pm 0.85$ & $2.35 \pm 0.96$ & 0.125 \\
\hline Monocyte $(\times 1000)$ & $0.55 \pm 0.19$ & $0.55 \pm 0.19$ & $0.55 \pm 0.23$ & 0.994 \\
\hline Basophil $(\times 1000)$ & $0.04(0.03-0.07)$ & $0.05(0.03-0.06)$ & $0.05(0.03-0.07)$ & 0.439 \\
\hline Hemoglobin (g/dL) & $12.54 \pm 1.15$ & $12.30 \pm 1.38$ & $12.27 \pm 1.44$ & 0.333 \\
\hline RDW (fL) & $38.11 \pm 4.11^{\mathrm{a}}$ & $35.41 \pm 3.27^{\mathrm{b}}$ & $34.86 \pm 3.16^{b}$ & $<0.001$ \\
\hline Platelet $(\times 1000)$ & $253.92 \pm 62.73$ & $243.40 \pm 55.17$ & $247.48 \pm 61.22$ & 0.553 \\
\hline MPV (fL) & $9.80 \pm 1.47$ & $9.64 \pm 1.35$ & $9.37 \pm 1.59$ & 0.170 \\
\hline PDW (fL) & $12.1(10.3-17.2)$ & $11.95(10.3-16.5)$ & $12.6(10.9-18.5)$ & 0.197 \\
\hline Monocyte/HDL Ratio & $8.33(6.48-10.86)$ & $7.67(5.76-10.36)$ & $8.36(6.35-11.61)$ & 0.334 \\
\hline
\end{tabular}

\section{Table 2. Results of linear regression analysis performed to determine factors effective on monocyte count}

\begin{tabular}{|lcccccccc|}
\hline & Unstandardized $\boldsymbol{\beta}$ & Standard Error & Standardized $\boldsymbol{\beta}$ & $\mathbf{t}$ & $\mathbf{p}$ & \multicolumn{2}{c|}{ 95.0\% Confidence Interval for $\boldsymbol{\beta}$} \\
\hline (Constant) & 0.456 & 0.192 & & 2.380 & 0.018 & 0.079 & 0.833 \\
Age & -0.004 & 0.003 & -0.085 & -1.317 & 0.189 & -0.009 & 0.002 \\
BMI & -0.001 & 0.002 & -0.033 & -0.488 & 0.626 & -0.006 & 0.003 \\
Triglyceride & 0.000 & 0.000 & -0.038 & -0.597 & 0.551 & -0.001 & 0.000 \\
HDL & 0.000 & 0.001 & 0.032 & 0.505 & 0.614 & -0.001 & 0.002 \\
Total cholesterol & 0.000 & 0.000 & 0.058 & 0.906 & 0.366 & 0.000 & 0.001 \\
Neutrophil & 0.008 & 0.005 & 0.098 & 1.568 & 0.118 & -0.002 & 0.018 \\
RDW & 0.004 & 0.004 & 0.069 & 1.075 & 0.283 & -0.003 & 0.011 \\
\hline
\end{tabular}

Table 3. Results of linear regression analysis performed to determine factors effective on HDL

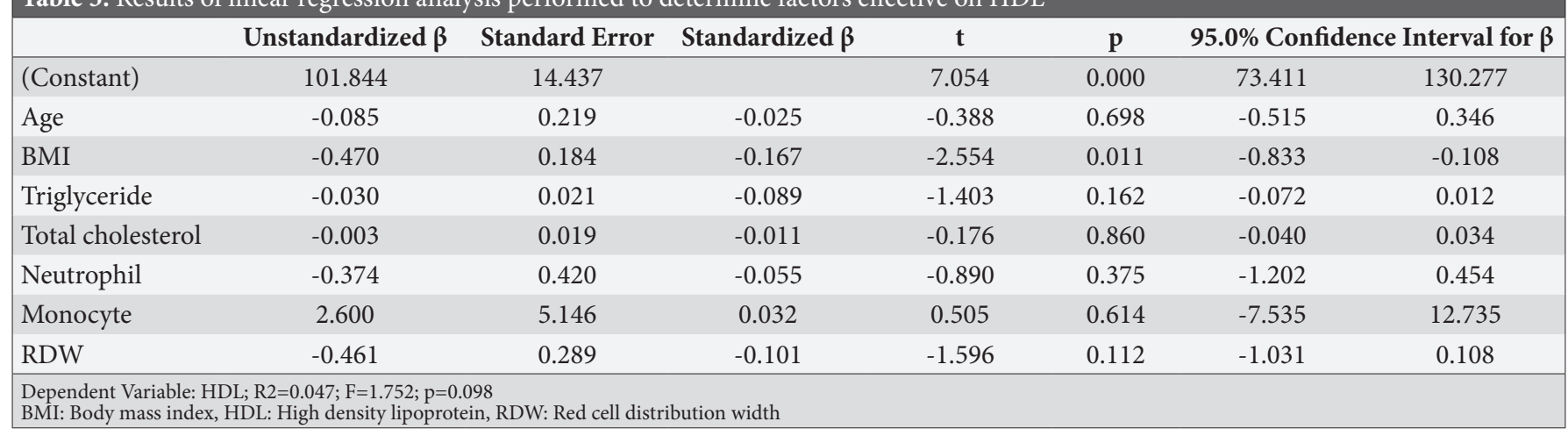




\section{DISCUSSION}

PCOS is an endocrine disorder with low-grade inflammation. In this study, in which various laboratory results were examined in PCOS, it was determined that MHR value was not affected by obesity or the presence of PCOS. Neutrophil count, as an inflammation marker, was found to be lower and RDW was higher in both PCOS groups compared to controls. In addition, triglyceride and total cholesterol were found to be elevated and HDL levels were decreased in the overweight/obese PCOS group. The results of the current study indicate that, despite causing adverse changes in lipid profile and inflammatory parameters, MHR cannot be utilized as a parameter that can quantify the relationship between these changes -even in the presence of obesity.

The presence of inflammation can be determined by examining different markers. Many different studies have shown the relationship between inflammation and increased monocyte count and decreased HDL (11-13). Studies that were based on the hypothesis of persistent inflammation in PCOS have shown an increase in the level of MHR as a result of an increase in monocyte count and a decrease in HDL, especially in obese individuals with PCOS. Usta et al. (18) reported that both monocyte count and MHR levels were significantly higher in PCOS cases compared to healthy women. They reported that these two variables were significant in terms of predicting inflammation in PCOS cases regardless of other risk factors. In addition, the obese control group, non-obese PCOS cases and obese PCOS cases were shown to have a significantly higher MHR value compared to the nonobese control group. In another study, Cakmak et al. (17) also showed that the MHR value was significantly higher in PCOS cases compared to healthy women. When compared to patients with PCOS who also had metabolic syndrome, women with PCOS who did not have metabolic syndrome and healthy controls were shown to have significantly lower MHR values. In a study examining monocyte activation and HDL function in healthy women and PCOS cases, Tedesco et al. (19) reported that macrophage response significantly decreased in PCOS cases compared to healthy controls. Furthermore, serum cholesterol efflux capacity (which is an indicator of HDL activity) was reportedly impaired in PCOS. Although the increased inflammation markers (including MHR) in patients with PCOS have been shown in previous studies, this relationship could not be shown in our study. It is possible that the lack of patients' medication data in the current study may have caused misinterpretation; however, a considerable effect that could alter the outcomes in such a large group is rather improbable.
When the neutrophil level was examined as a measure of inflammation in our study, it was found that neutrophil levels were significantly lower in both the normoweight and overweight PCOS groups compared to the control group. In a study on this subject, Herlihy et al. (20) reported that the frequency of elevated neutrophil levels were higher in patients with PCOS compared to controls $(14 \%$ vs. $4 \%)$. In addition, they reported that neutrophil levels correlated positively with body fat mass. In many other studies, it has been shown that, when compared to healthy women, PCOS cases have an increased neutrophil level with adjustments for age and BMI (2123). The neutrophil level has been shown to decrease with metformin and flutamide therapy and the use of various anti-inflammatory drugs $(21,24)$. In our study, the drugs used by the patients for therapeutic purposes were not evaluated. Such drugs used in the treatment of PCOS cases may have affected neutrophil levels. In addition, the older age of the control group could also have contributed to this result.

Red blood cell distribution width, another widelyaccepted marker of chronic inflammation, has been evaluated for many diseases. It has been reported that RDW value significantly changes in diseases that progress with chronic inflammation and oxidative stress $(25,26)$. Since PCOS is a disease with chronic low-grade inflammation at its basis, RDW value is expected to be affected. Consistent with this expectation, Yilmaz et al. (27) reported that RDW value was significantly higher in PCOS cases compared to healthy controls. In addition, the study stated that a threshold for RDW (>12.5) could be used as a cut-off to identify PCOS. Similarly, Calan et al. (28) showed an increase in RDW in PCOS patients. In addition, RDW has been shown to increase significantly as a result of weight gain (29). In our study, contrary to the studies in the literature, it was determined that RDW value was higher in healthy women compared to the PCOS groups, and that there was no significant difference between the PCOS groups with regard to BMI-based groups. Parallel to our interpretation in the output of other inflammation markers, this may be due to the fact that additional inflammatory conditions were not evaluated in our study, and that the control group consisted of older individuals.

Regardless of other chronic/acute diseases, unhealthy weight gain is an undesirable condition that increases the level of total cholesterol and LDL and lowers the level of HDL; thereby creating a basis for different chronic diseases. When the studies conducted with PCOS cases on this subject were examined, Usta et al. (18) reported that total cholesterol and LDL levels were higher and HDL levels were lower in obese PCOS cases compared to obese controls. Cakmak et al. (17) reported that the 
level of triglycerides was higher in patients with PCOS who had metabolic syndrome compared to those without metabolic syndrome, while the total cholesterol and LDL values were similar between the groups. In our study, triglyceride and total cholesterol values were higher and HDL values were lower in the overweight/obese PCOS group. Furthermore, it was thought that high levels of triglycerides, total cholesterol and low HDL levels identified in the overweight/obese PCOS group could be attributed to increased BMI rather than PCOS. However, it is also possible that these alterations could indicate an underlying change in endothelial function, since triglycerides have been suggested to be a marker of endothelial (dys)function in healthy individuals and also patients with metabolic syndrome (30).

It is an important limitation that our study has a retrospective design. Acute infectious diseases and the medication use of patients could not be assessed, and the lack of these evaluations may have affected the outcomes of our study -especially if the distribution of these characteristics were unequal between the groups. Also, the age of the control group was higher, which may be an important factor that altered results.

\section{CONCLUSION}

It was determined that MHR value was similar in healthy women and PCOS cases grouped on the basis of BMI. Although the importance of MHR was emphasized in predicting PCOS cases in previous studies, it was concluded that this relationship was not significant in our study. These results may indicate an underlying difference in patient characteristics, but may have also been caused by the baseline differences between groups. Nevertheless, it appears that MHR has little value in the clinical assessment of patients with PCOS, regardless of obesity. In addition, interestingly, neutrophil was lower and RDW was higher in PCOS cases. Although the study has various limitations, considering the number of studies on this subject, it can be said that it will be useful to clarify this issue with future studies.

\section{ETHICAL DECLARATIONS}

Ethics Committee Approval: Ethics committee approval was obtained from the Ethics Committee of Health Sciences University Adana City Training and Research Hospital (Approval no: 878, Date: 2020/05.20).

Informed Consent: Because the study was designed retrospectively, no written informed consent form was obtained from patients.

Referee Evaluation Process: Externally peer-reviewed.

Conflict of Interest Statement: The authors have no conflicts of interest to declare.
Financial Disclosure: The authors declared that this study has received no financial support.

Author Contributions: All of the authors declare that they have all participated in the design, execution, and analysis of the paper, and that they have approved the final version.

\section{REFERENCES}

1. Escobar-Morreale HF. Polycystic ovary syndrome: definition, aetiology, diagnosis and treatment. Nature Reviews Endocrinology 2018; 14: 270.

2. Bozdag G, Mumusoglu S, Zengin D, Karabulut E, Yildiz BO. The prevalence and phenotypic features of polycystic ovary syndrome: a systematic review and meta-analysis. Hum Reprod 2016; 31: 2841-55.

3. Cheang KI, Huszar JM, Best AM, Sharma S, Essah PA, Nestler JE. Long-term effect of metformin on metabolic parameters in the polycystic ovary syndrome. Diabetes and Vascular Disease Research 2009; 6: 110-9.

4. Randeva HS, Tan BK, Weickert MO, et al. Cardiometabolic aspects of the polycystic ovary syndrome. Endocr Rev 2012; 33: 812-41.

5. Sathyapalan T, Atkin SL. Mediators of inflammation in polycystic ovary syndrome in relation to adiposity. Mediators Inflamm 2010; 2010

6. Wołczyński S, Zgliczyński W. Abnormalities of the menstrual cycle. Large Interna-Endocrinology 2 nd edition Medical Tribune Poland, Warsaw 2012: 561-7.

7. Group REaSPCW. Revised 2003 consensus on diagnostic criteria and long-term health risks related to polycystic ovary syndrome (PCOS). Hum Reprod 2004; 19: 41-7.

8. Kharitonenkov A, Adams AC. Inventing new medicines: the FGF21 story. Molecular metabolism 2014; 3: 221-9.

9. Bednarska S, Siejka A. The pathogenesis and treatment of polycystic ovary syndrome: What's new. Adv Clin Exp Med 2017; 26: 359-67.

10. Patel S. Polycystic ovary syndrome (PCOS), an inflammatory, systemic, lifestyle endocrinopathy. The Journal of steroid biochemistry and molecular biology 2018; 182: 27-36.

11. Auffray C, Sieweke MH, Geissmann F. Blood monocytes: development, heterogeneity, and relationship with dendritic cells. Annu Rev Immunol 2009; 27.

12. Parthasarathy S, Barnett J, Fong LG. High-density lipoprotein inhibits the oxidative modification of low-density lipoprotein. Biochimica et Biophysica Acta (BBA)-Lipids and Lipid Metabolism 1990; 1044: 275-83.

13. Westerterp M, Gourion-Arsiquaud S, Murphy AJ, et al. Regulation of hematopoietic stem and progenitor cell mobilization by cholesterol efflux pathways. Cell stem cell 2012; 11: 195-206.

14. Kaplan I, Kaplan M, Abacioglu O, Yavuz F, Saler T. Monocyte/ HDL ratio predicts hypertensive complications. Bratisl Lek Listy 2020; 121: 133-6.

15. Yakar H, Kanbay A. Could monocyte level/HDL cholesterol ratio predict cardiovascular diseases in patients with COPD? Niger J Clin Pract 2020; 23: 450.

16. Sirakaya E, Duru Z, Kuçuk B, Duru N. Monocyte to high-density lipoprotein and neutrophil-to-lymphocyte ratios in patients with acute central serous chorioretinopathy. Indian J Ophthalmol 2020; 68: 854 .

17. Dincgez Cakmak B, Dundar B, Ketenci Gençer F, Aydin BB, Yildiz DE. TWEAK and monocyte to HDL ratio as a predictor of metabolic syndrome in patients with polycystic ovary syndrome. Gynecol Endocrinol 2019; 35: 66-71. 
18. Usta A, Avci E, Bulbul CB, Kadi H, Adali E. The monocyte counts to HDL cholesterol ratio in obese and lean patients with polycystic ovary syndrome. Reprod Biol Endocrinol 2018; 16: 34.

19. Tedesco S, Adorni MP, Ronda N, et al. Activation profiles of monocyte-macrophages and HDL function in healthy women in relation to menstrual cycle and in polycystic ovary syndrome patients. Endocrine 2019; 66: 360-9.

20. Herlihy A, Kelly R, Hogan J, O'connor N, Farah N, Turner M. Polycystic ovary syndrome and the peripheral blood white cell count. J Obstet Gynaecol 2011; 31: 242-4.

21. Ibáñez L, Jaramillo AM, Ferrer A, De Zegher F. High neutrophil count in girls and women with hyperinsulinaemic hyperandrogenism: normalization with metformin and flutamide overcomes the aggravation by oral contraception. Hum Reprod 2005; 20: 2457-62.

22. Thomann R, Rossinelli N, Keller U, et al. Differences in lowgrade chronic inflammation and insulin resistance in women with previous gestational diabetes mellitus and women with polycystic ovary syndrome. Gynecol Endocrinol 2008; 24: 199206.

23. Orio Jr F, Palomba S, Cascella T, et al. The increase of leukocytes as a new putative marker of low-grade chronic inflammation and early cardiovascular risk in polycystic ovary syndrome. The Journal of Clinical Endocrinology \& Metabolism 2005; 90: 2-5.

24. Diamanti-Kandarakis E, Paterakis T, Alexandraki K, et al. Indices of low-grade chronic inflammation in polycystic ovary syndrome and the beneficial effect of metformin. Hum Reprod 2006; 21: 1426-31.

25. Tsuboi S, Miyauchi K, Kasai T, et al. Impact of red blood cell distribution width on long-term mortality in diabetic patients after percutaneous coronary intervention. Circ J 2013; 77: 45661.

26. Vayá A, Carmona P, Badia N, Hernandez-Mijares A, Bautista D. Association between high red blood cell distribution width and metabolic syndrome. Influence of abdominal obesity. Clin Hemorheol Microcirc 2011; 47: 75-7.

27. Yilmaz Ö, Mehmet C, Kelekci S, Temur M. Association between red blood cell distribution width and polycystic ovary syndrome. Endocr Res 2015; 40: 181-7.

28. Calan M, Yilmaz O, Kelekci S. Is elevated red blood cell distribution width a new risk factor for polycystic ovary syndrome? 16th European Congress of Endocrinology: BioScientifica; 2014.

29. Fujita B, Strodthoff D, Fritzenwanger M, et al. Altered red blood cell distribution width in overweight adolescents and its association with markers of inflammation. Pediatr Obes 2013; 8: 385-91.

30. Kajikawa M, Higashi Y. Triglycerides and endothelial function: molecular biology to clinical perspective. Curr Opin Lipidol 2019; 30: 364-9. 